\title{
A decade of molecular genetic testing for MODY: a retrospective study of utilization in The Netherlands
}

\author{
Stephanie S Weinreich ${ }^{\star}, 1$, Astrid Bosma ${ }^{1}$, Lidewij Henneman ${ }^{1}$, Tessel Rigter ${ }^{1}$, Carla MJ Spruijt ${ }^{2}$, \\ Anneliese JEMA Grimbergen ${ }^{2}$, Martijn H Breuning ${ }^{2}$, Eelco JP de Koning ${ }^{3}$, Monique Losekoot ${ }^{2}$ \\ and Martina C Cornel ${ }^{1}$
}

Genetic testing for maturity-onset diabetes of the young (MODY) may be relevant for treatment and prognosis in patients with usually early-onset, non-ketotic, insulin-sensitive diabetes and for monitoring strategies in non-diabetic mutation carriers. This study describes the first 10 years of genetic testing for MODY in The Netherlands in terms of volume and test positive rate, medical setting, purpose of the test and age of patients tested. Some analyses focus on the most prevalent subtype, HNF1A MODY. Data were retrospectively extracted from a laboratory database. In total, 502 individuals were identified with a pathogenic mutation in HNF4A, GCK or HNF1A between 2001 and 2010. Although mutation scanning for MODY was used at an increasing rate, cascade testing was only used for one relative, on average, per positive index patient. Testing for HNF1A MODY was mostly requested by internists and paediatricians, often from regional hospitals. Primary care physicians and clinical geneticists rarely requested genetic testing for HNF1A MODY. Clinical geneticists requested cascade testing relatively more often than other health professionals. A substantial proportion (currently $29 \%$ ) of HNF1A MODY probands was at least 40 years old at the time of testing. In conclusion, the number of individuals genetically tested for MODY so far in The Netherlands is low compared with previously predicted numbers of patients. Doctors' valuation of the test and patients' and family members' response to (an offer of) genetic testing on the other hand need to be investigated. Efforts may be needed to develop and implement translational guidelines.

European Journal of Human Genetics (2015) 23, 29-33; doi:10.1038/ejhg.2014.59; published online 16 April 2014

\section{INTRODUCTION}

Maturity-onset diabetes of the young (MODY) is the most common monogenic type of diabetes, estimated to account for up to $1.8 \%$ of patients with diabetes. ${ }^{1,2}$ MODY is usually characterized by an early-onset of diabetes (often before the age of 40 years), no requirement for insulin at diagnosis, no signs of autoimmunity or insulin resistance and autosomal dominant inheritance. ${ }^{3}$ MODY is a clinically heterogeneous group of disorders caused by $\beta$-cell dysfunction. At the molecular genetic level, MODY is defined by mutations in different genes, of which at least 13 are known. ${ }^{4}$ The most common forms are HNF1A (MODY3, OMIM ${ }^{\star} 600281$ ), GCK (MODY2, OMIM ${ }^{\star}$ 138079) and HNF4A (MODY1, OMIM $\left.{ }^{\star} 600281\right) .{ }^{1}$ The various genetic subtypes differ in clinical manifestation: age of onset, pattern of hyperglycemia, response to treatment and associated extrapancreatic manifestations. ${ }^{5}$

Molecular genetic testing in symptomatic patients has several benefits. Diagnosis may have implications for treatment such as the preferred use of sulphonylurea derivatives in HNF1A MODY or stopping insulin treatment in GCK MODY. ${ }^{6}$ The diagnosis of GCK MODY is considered important in relation to pregnancy as it helps to understand the presence or absence of macrosomia and may direct therapeutic decisions. ${ }^{5,7}$ A correct genetic diagnosis also has implications for anticipation of complications, as microvascular complications are much rarer in GCK than in HNF1A and HNF4A
MODY. ${ }^{8}$ Once a patient has received a genetic diagnosis of MODY, predictive or pre-symptomatic genetic testing can be offered to family members to confirm or exclude a genetic predisposition (so-called cascade testing). In the case of GCK MODY, genetic testing of family members is usually a second-tier test after screening for fasting blood glucose. ${ }^{5}$ Family members identified with a mutation in a MODY-associated gene can be offered appropriate monitoring strategies. ${ }^{5}$ Taken together, molecular genetic testing can benefit both symptomatic and pre-symptomatic patients. However, as with any genetic test, the benefits must be balanced with the costs and possible disadvantages (eg, psychosocial impact and insurancerelated issues). ${ }^{9}$

In The Netherlands, patients with type 2 diabetes are most often cared for by general practitioners (GPs) with one of the main goals being prevention or delay of complications. ${ }^{10}$ GPs and their support staff provide this level of care, in line with the traditional gate-keeping function of GPs in The Netherlands. If treatment goals are not achieved or patients develop moderate-to-severe diabetes-related complications patients are referred to hospitals (secondary care). ${ }^{11}$ Also patients with type 1 diabetes are generally cared for in hospitals. Specialist care can be provided in a hospital setting or in extramural diabetes centres.

Since 2001, the Laboratory for Diagnostic Genome Analysis at the Department of Clinical Genetics at Leiden University Hospital has

${ }^{1}$ Department of Clinical Genetics, Section of Community Genetics, EMGO Institute for Health and Care Research, VU University Medical Center, Amsterdam, The Netherlands; ${ }^{2}$ Department of Clinical Genetics, Leiden University Medical Center, Leiden, The Netherlands; ${ }^{3}$ Department of Endocrinology and Department of Nephrology, Leiden University Medical Center, Leiden, The Netherlands

${ }^{*}$ Correspondence: Dr SS Weinreich, Department of Clinical Genetics, Section of Community Genetics, EMGO Institute for Health and Care Research, VU University Medical Center, PO Box 7057, BS7 D450, 1007 MB Amsterdam, The Netherlands. Tel: +31 20444 8686; Fax: +31 20 444 8665; E-mail: s.weinreich@vumc.nl

Received 9 August 2013; revised 10 March 2014; accepted 13 March 2014; published online 16 April 2014 
provided genetic testing for MODY for all of The Netherlands. Occasionally, testing is also provided in response to requests from abroad. A few other centres in The Netherlands also offer genetic testing for other forms of diabetes. ${ }^{12}$ Previously, it was estimated that there are about 20000 MODY patients in The Netherlands. ${ }^{13,14}$ In 2005, 65 patients had been identified in Leiden ${ }^{14}$ but it is unclear how many patients have been diagnosed since then. If the availability of molecular genetic testing has not identified the expected numbers of patients, the reasons for this require clarification. The first aim of this study is to describe the current use of genetic testing for MODY in The Netherlands in terms of volume and test positive rate, medical setting (physician characteristics and type of institution requesting a test), purpose of the test (diagnostic or predictive) and age of patients tested. A second aim is to explore some historical trends. We include the three most common MODY subtypes and highlight details of HNF1A MODY as an example. HNF1A MODY has the highest prevalence, ${ }^{15}$ genetic diagnosis is relevant for family members and referral practices for $H N F 1 A$ are expected to be similar to those for GCK and HNF4A. Details are compared between two early and two recent years since the introduction of molecular genetic testing.

To our knowledge, this is the first study to both quantify and characterize the use of genetic testing for MODY in a health-care system. It represents a first step in developing suggestions for better practice.

\section{MATERIALS AND METHODS}

\section{Technical features of the test}

Since 2001, sequence analysis of the coding region including intron-exon boundaries of GCK, HNF1A and HNF4A has been performed using standard procedures $^{14}$ (details available upon request). Since 2008, multiplex ligationdependent probe amplification has been used for the detection of deletions and duplications (MRC-Holland, Amsterdam, The Netherlands, kit P241).

The physician decides whether mutation analysis is indicated. For reasons of efficiency mutation, scanning is usually performed simultaneously for the three most commonly affected genes (HNF1A, GCK, HNF4A). Mutation analysis of other genes involved in MODY is performed upon specific request of the referring physician.

\section{Informed consent and ethics committee approval}

The Medical Ethics Committees of VU University Medical Center and Leiden University Medical Center deemed this research to be beyond the scope of the Medical Research Involving Human Subjects Act.

\section{Database and data extraction}

The oracle-based, home-made, Laboratory Information System from the Leiden Laboratory for Diagnostic Genome Analysis was used to extract the patient and referring physician information for this study. Several questions (SQL-queries, structured query language) were formulated and data were extracted anonymously.

Anonymous data files were checked for logical consistency by one of the authors (SSW). In consensus with another author (ML), corrections were made, eg, the exclusion of non-Dutch or non-diagnostic samples (eg, quality controls).

\section{Categorization}

Paediatricians and paediatric endocrinologists were categorized together. Internists and endocrinologists were also categorized together. The category 'other' physicians includes trainees and physicians with other or unknown specialties.

The age of patients tested for MODY was categorized as $<12,12-17,18-24$, $25-39$ and $\geq 40$ years. These divisions were motivated by distinctions in medical law regarding informed consent for genetic testing $(<12,12-17$ and $18+$ years), by the fact that MODY was historically considered to manifest by age 25 years, and to illustrate a group ( $\geq 40$ years), which very likely already had children before becoming aware of a risk for transmitting a pathogenic mutation for MODY.

\section{Statistical analyses}

Probands' age at testing for HNF1A was compared for two time periods with the independent samples $t$-test, using IBM SPSS Statistics for Windows, version 20.0 (IBM Corp, Armonk, NY, USA).

\section{RESULTS}

\section{Volume of testing and detection rate}

Figure 1 shows the number and type of tests for MODY performed between 2001 and 2010. Mutation scanning has shown continued growth over the years, with over 212 requests for mutation scanning in 2010. In total, 1319 mutation scans were performed for HNF1A, GCK or HNF4A, nearly always in multiplex.

The test positive rate (mutation detection ratio, diagnostic sensitivity) for multiplex testing for HNF1A, GCK or HNF4A has been fairly constant (Figure 1). For example, in the years 2003 and 2004 , the average test positive rate was $25 \%$ ( 45 pathogenic mutations in 183 patients), whereas in 2009 and 2010, it was 22\% (91 pathogenic mutations in 422 patients). Figure 1 also shows the rate of cascade testing for known mutations in family members over the years. Between 2001 and 2010, 296 tests were performed for known mutations in family members (48 tests in 2010). Over the 10-year period studied, for each genetically diagnosed index patient about one family member, on average, had a cascade test. Altogether, between 2001 and 2010, a total of 502 persons was identified with a pathogenic MODY mutation (222 HNF1A, 204 GCK and 76 HNF4A).

\section{Physicians requesting genetic testing for HNF1A MODY}

Figure 2a shows the specialty of physicians who requested a test for HNF1A MODY, in an early and recent period since testing became available. Each physician was counted once in the year that he or she requested a test for any patient(s). Most referring physicians were internists/endocrinologists and paediatricians/paediatric endocrinologists. Requests from clinical geneticists were rare and from GPs they were very exceptional.

Because cascade testing for family members is not widely implemented, an analysis was done to see if its use is restricted to certain specialties. Table 1 shows that in 2009 and 2010, cascade testing for HNF1A most often originated from paediatricians in absolute numbers (13 requests). Clinical geneticists were relatively most likely to request cascade testing ( $24 \%$ of their total requests).

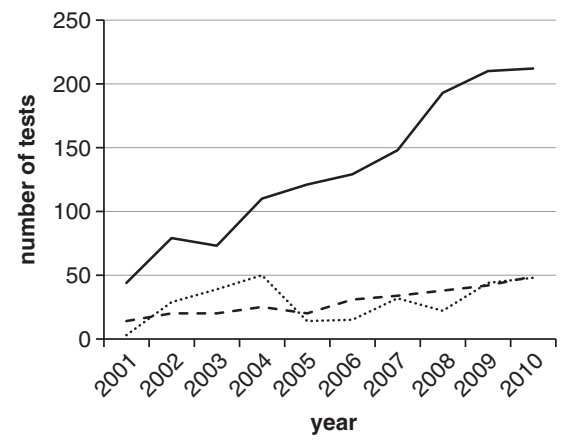

Figure 1 Molecular genetic testing for HNF4A, GCK and HNF1A MODY over the years 2001-2010. Solid line: mutations scans (nearly always multiplex testing for HNF4A, GCK and HNF1A). Dashed line: positive mutation scans. Dotted line: tests for known mutations (cascade testing) in HNF4A, GCK and HNFIA. 

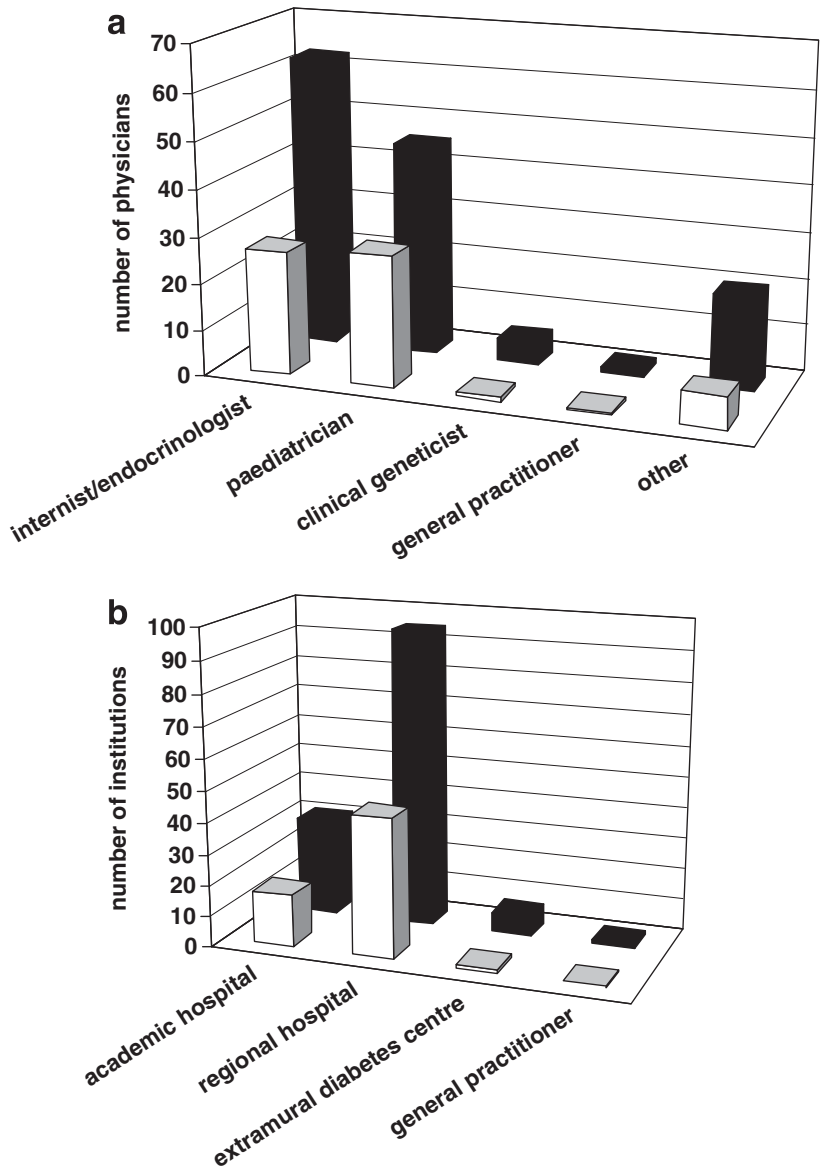

Figure 2 Characteristics of requesters of genetic testing for HNF1A in early (2003-2004) and later (2009-2010) years of test availability. Light bars show averages for 2003-2004; dark bars show averages for 2009-2010. (a) Specialty of requesting physicians. Paediatricians include paediatric endocrinologists. Category 'other' includes trainee, other or unknown physicians. Each physician was counted once in the year that he/she requested a test for any patient(s). (b) Type of medical institution requesting testing. Multiple referring physicians from the same institution are counted separately.

Table 1 Requests for mutation scanning vs cascade testing for HNF1A per specialty in recent years of test availability (2009 and 2010)

\begin{tabular}{lccc}
\hline Specialty & $\begin{array}{c}\text { Total } \\
\text { requests } \\
\text { for testing }\end{array}$ & $\begin{array}{c}\text { No. of } \\
\text { cascade } \\
\text { tests }\end{array}$ & $\begin{array}{c}\text { Rate of } \\
\text { cascade } \\
\text { testing (\%) }\end{array}$ \\
\hline Internist/endocrinologist & 225 & 11 & 5 \\
Paediatrician/paediatric endocrinologist & 163 & 13 & 8 \\
Clinical geneticist & 21 & 5 & 24 \\
Other $^{\text {b }}$ & 42 & 0 & 0 \\
Total $^{\text {ata }}$ & 451 & 29 & \\
\hline
\end{tabular}

a Mutation scanning plus cascade testing, 2009 and 2010 taken together.

bIncluding 5 requests from general practitioners and 37 requests from trainee, other or unknown physicians.

Medical setting from which testing is requested

Next, a comparison was made of the medical institutions where the referring physicians for HNF1A MODY testing are working (Figure $2 \mathrm{~b}$ ). The early years of genetic testing for MODY and the
Table 2 Age of probands tested for HNF1A

\begin{tabular}{lcccc}
\hline Age (years) & \multicolumn{2}{c}{2003 and 2004} & 2009 and 2010 \\
\hline$<12$ & 37 & $20 \%$ & 68 & $16 \%$ \\
$12-17$ & 42 & $23 \%$ & 83 & $20 \%$ \\
$18-24$ & 14 & $8 \%$ & 42 & $10 \%$ \\
$25-39$ & 53 & $29 \%$ & 108 & $26 \%$ \\
$\geq 40$ & 37 & $20 \%$ & 121 & $29 \%$ \\
Total $^{\text {a }}$ & 183 & $100 \%$ & 422 & $100 \%$ \\
\hline
\end{tabular}

aPercentages in the last column add up to $>100 \%$ due to rounding.

recent period both show a higher number of referring physicians from regional hospitals than academic hospitals. A small number of referring physicians were from extramural diabetes centres. Primary care physicians rarely requested testing for HNF1A MODY.

\section{Age of patients tested for HNF1A}

A historic diagnostic criterion for MODY is hyperglycemia before the age of 25 years in at least one family member. ${ }^{3}$ Because genetic testing first became available in 2001, this was the first opportunity for MODY patients of any age to obtain a genetic diagnosis. We hypothesized that as time went on, the average age at genetic testing for MODY might have dropped.

To explore this question, using HNF1A MODY as an example, probands from the early and late periods since testing became available (2003-2004 vs 2009-2010) were compared. The average age at testing in fact rose slightly from 25.7 to 29.2 years (independent samples $t$-test; difference $=-3.5 ; 95 \% \quad \mathrm{CI}=-6.6$ to -0.50 ; $P=0.022)$. To illustrate further details, probands were categorized by age (Table 2 ). Notably, a substantial proportion (20\% and $29 \%)$ of probands was over 40 years old at the time of testing in 2003-2004 and 2009-2010, respectively. Only about half the probands tested were under 25 years of age ( 51 and $46 \%$ ).

\section{DISCUSSION}

This study shows that while mutation scanning for MODY has been used at an increasing rate in The Netherlands during the first 10 years of its availability, cascade testing has only been used by one relative, on average, per positive index patient. Taking HNF1A as an example, requests for genetic testing typically come from internists/endocrinologists and paediatricians/paediatric endocrinologists working in regional and academic hospitals or extramural diabetes centres. GPs rarely request testing. Clinical geneticists request a higher proportion of cascade tests than other health-care providers. MODY testing targets people of a wide age range.

\section{Strengths and limitations of the study}

The compact size of The Netherlands, the clear cut three-tiered health-care system and the concentration of testing in one laboratory provide an accurate overview of how molecular genetic testing for MODY has been utilized in a population of 16 million people. We focused on the most prevalent form HNF1A MODY to illustrate characteristics of the physician and patient populations involved in genetic testing. The cumulative number of genetic tests for MODY was previously described for The Netherlands up to October 2003. ${ }^{14}$ We now provide insight into which practitioners in the Dutch healthcare system make use of MODY testing and we address changes over time. A limitation of this study is that clinical data were not considered. This would have given deeper insight into the characteristics besides age of patients selected for MODY testing. 


\section{Comparison with other studies}

This research demonstrated diversity in referral patterns for genetic testing for MODY in The Netherlands, at the level of medical specialty and the medical setting. An overview on use of genetic testing for MODY has also been published for the entire United Kingdom, covering the period 1996-2009. ${ }^{1}$ The UK study also found diversity in referral patterns, at the geographic level (country and/or region).

This study shows that in The Netherlands so far 502 individuals have been identified with a pathogenic mutation in HNF1A, GCK or $H N F 4 A$, including diabetic patients and diabetic and non-diabetic family members. This figure is equivalent to 30 per million (Netherlands' population in 2010 was 16.6 million $)^{16}$ and it can be compared with recent findings in other countries. A minimum population prevalence of 68-108 individuals with a MODY mutation per million was calculated from the areas with highest rates of MODY detection in the United Kingdom; ${ }^{1}$ this includes both diabetic patients as well as family members who may be pre-symptomatic. In addition, it is relevant to consider studies restricted to diabetic (symptomatic) patients. A study from Poland suggested a prevalence of 42-46 cases of monogenic diabetes per million children aged $0-18$ years, ${ }^{17}$ which can be extrapolated to 189-207 cases per million for the entire Dutch population assuming a normal lifespan for MODY patients (population ca 3.7 million children under 19 years).$^{18}$ Finally, a Slovakian study calculated a minimum prevalence of 20 cases per million of GCK MODY. ${ }^{19}$ If GCK accounts for about one-third of MODY as suggested by this study (raw data for Figure 1, not shown), the total prevalence of MODY for Slovakia can be extrapolated to about 60 cases per million. Taken together, the number of people in The Netherlands with a proven mutation in a MODY gene is half of the lowest minimum prevalence predicted by recent studies in other countries where genetic testing has been implemented, even including studies that considered symptomatic patients only. Notably, all of these studies have lower estimates than the prevalence predicted before implementation of genetic testing for MODY, ie, about 20000 patients for The Netherlands, ${ }^{13}$ equivalent to 1204 patients per million. The difference might be due to high costs or lack of perceived utility of genetic testing among physicians (especially for pre-symptomatic relatives of patients), reluctance to be tested among patients and/or pre-symptomatic family members (eg, because of fear of the potential consequences for mortgages, life- and health insurance), lack of translational guidelines, genetically unexplained cases which meet the clinical criteria for MODY and/or overestimation in the original figure.

The example of HNF1A shows that there is a wide age range for referring probands for molecular genetic testing (Table 2). This means that doctors who make referrals have to deal with a variety of clinical genetic issues. Paediatric health practitioners, for example, must tailor information and consent procedures for several, legally recognized categories of minors. Health-care practitioners treating adults will have to address the implications of genetic testing for reproductive choices as well as the impact of a genetic diagnosis for patients who already have children.

We did not find evidence that probands have been getting tested for HNF1A at a younger age since the introduction of molecular genetic testing for MODY. A substantial proportion of probands described in this study was over 40 years old at the time of testing, in accordance with recent findings in a French study. ${ }^{20}$ An explanation could be that diabetes can be undiagnosed for a long time in MODY patients. Or MODY can be associated with mild, non-progressive symptoms into middle age, ${ }^{21}$ finally leading to genetic testing. It could also mean that awareness of MODY is still growing among physicians, and that a backlog of older, symptomatic patients is being assessed for a genetic diagnosis.

This study shows that genetic testing for MODY in The Netherlands is firmly in the sphere of medical specialists, rather than primary care. A similar pattern has been suggested for the United Kingdom. ${ }^{1}$ In The Netherlands, most patients with type 2 diabetes, and thus probably most misdiagnosed MODY patients, are cared for by GPs and their support staff. Policy researchers suggest that genetic testing or screening for common disorders including diabetes might be added to the existing procedures in primary care, through cooperation between primary and specialist care. ${ }^{22}$ This may require help from intermediary organizations, as for example in a screening programme for familial hypercholesterolemia. ${ }^{22,23}$

\section{Directions for future research}

To better understand the current use of genetic testing for MODY, several approaches could be taken. Qualitative research can reveal doctors' valuation of the test on the one hand and patients' and family members' response to an offer of genetic testing on the other hand (early experiences have been described previously). ${ }^{24}$ The rather low rate of cascade testing found in this study might be due to a preference for phenotypic monitoring of non-diabetic relatives, as recently recommended, ${ }^{25}$ or there may be financial or other concerns related to cascade testing. Furthermore, current use of cascade testing could be studied in more detail if clinical data were coupled to the laboratory data. This would shed light on the number of presymptomatic and asymptomatic family members tested in relation to the number of symptomatic family members tested, the latter perhaps previously misdiagnosed with another form of diabetes. Finally, discussion is needed on whether to adapt current practices and if so, who should take the initiative. In the United Kingdom, it was recently shown that genetic funders perceive genetic testing for MODY as mainstream medicine, whereas diabetes commissioners see it as specialist genetics. ${ }^{26}$ In The Netherlands, it may also be a challenge to bridge this gap.

\section{CONFLICT OF INTEREST}

The authors declare no conflict of interest.

\section{ACKNOWLEDGEMENTS}

We thank TCTEF van Cronenburg (Leiden University Medical Center) for her help in extracting the data from the Laboratory Information System. The study was funded by The Netherlands Genomics Initiative (Project no. CMSB/CSG I.2h) as part of the programme of the Centre for Society and the Life Sciences and the Centre for Medical Systems Biology in The Netherlands. An abstract of this work was presented at the European Society for Human Genetics 2013, P04.87

1 Shields BM, Hicks S, Shepherd MH, Colclough K, Hattersley AT, Ellard S: Maturity-onset diabetes of the young (MODY): how many cases are we missing? Diabetologia 2010; 53: 2504-2508.

2 Ledermann HM: Is maturity onset diabetes at young age (MODY) more common in Europe than previously assumed? Lancet 1995; 345: 648.

3 Vaxillaire M, Froguel P: Genetic basis of maturity-onset diabetes of the young. Endocrinol Metab Clin North Am 2006; 35: 371-384.

4 Bonnefond A, Philippe J, Durand E et al: Whole-exome sequencing and high throughput genotyping identified KCNJ11 as the thirteenth MODY gene. PLoS One 2012; 7: e37423.

5 Murphy R, Ellard S, Hattersley AT: Clinical implications of a molecular genetic classification of monogenic beta-cell diabetes. Nat Clin Pract Endocrinol Metab 2008; 4: 200-213 
6 Pearson ER, Starkey BJ, Powell RJ, Gribble FM, Clark PM, Hattersley AT: Genetic cause of hyperglycaemia and response to treatment in diabetes. Lancet 2003; 362: 1275-1281.

7 Spyer G, Macleod KM, Shepherd M, Ellard S, Hattersley AT: Pregnancy outcome in patients with raised blood glucose due to a heterozygous glucokinase gene mutation. Diabet Med 2009; 26: 14-18.

8 Naylor R, Philipson LH: Who should have genetic testing for MODY? Clin Endocrinol (Oxf) 2011; 75: 422-426.

9 Shepherd M: Genetic testing in maturity onset diabetes of the young (MODY) - practical guidelines for professionals. Pract Diab Int 2003; 20: 108-110.

10 Baan CA, Schoemaker CG, Jacobs-van der Bruggen MAM et al: Diabetes tot 2025. 2009; Available from: http://www.rivm.nl/bibliotheek/rapporten/260322004.html. Accessed on 28 June 2013. p 67.

11 Hamberg-van Reenen HH: (RIVM): Diabetes mellitus: Hoe zijn preventie en zorg georganiseerd? In: Volksgezondheid Toekomst Verkenning, Nationaal Kompas Volksgezondheid. 2011; Available from: http://www.nationaalkompas.nl/gezondheiden-ziekte/ziekten-en-aandoeningen/endocriene-voedings-en-stofwisselingsziektenen-immuniteitsstoornissen/diabetes-mellitus/diabetes-mellitus-preventie-en-zorg/ Accessed on 28 June 2013.

12 Dutch National Board for DNA-diagnostics: Overzicht DNA diagnostiek Nederland 2012, Available from: http://www.dnadiagnostiek.nl/zoekenStart_nl.php. Accessed on 28 June 2013.

13 Maassen JA, Lemkes HH, Losekoot M: [From gene to disease; 'maturity-onset diabetes of the young' (MODY), monogenetic inheritable forms of diabetes mellitus]. Ned Tijdschr Geneeskd 2001; 145: 1352-1353.

14 Losekoot M, Broekman AJ, Breuning MH, de Koning EJP, Romijn JA, Maassen JA: [Molecular diagnosis on indication of maturity onset diabetes of the young; results from 184 patients]. Ned Tijdschr Geneeskd 2005; 149: 139-143.

15 Estalella I, Rica I, Perez de Nanclares G et al: Mutations in GCK and HNF-1alpha explain the majority of cases with clinical diagnosis of MODY in Spain. Clin Endocrinol (Oxf) 2007; 67: 538-546.
16 Statistics Netherlands. Population; key figures. 2013; Available from: http://statline. cbs.nl/StatWeb/publication/?DM=SLEN\&PA=37296ENG\&D1=0-2,19-21,23-27,52-55, 57-68\&D2 $=60 \& L A=E N \& V W=T$. Accessed on 28 June 2013.

17 Fendler W, Borowiec M, Baranowska-Jazwiecka A et al: Prevalence of monogenic diabetes amongst Polish children after a nationwide genetic screening campaign. Diabetologia 2012; 55: 2631-2635.

18 Statistics Netherlands Bevolking; geslacht, leeftijd en burgerlijke staat, 1 januari. 2013; Available from: http://statline.cbs.nl/StatWeb/publication/?DM=SLNL\&PA= 7461BEV\&D1=a\&D2=a\&D3=0-100\&D4=0,(I-2)-I\&VW=T. Accessed on 28 June 2013

19 Valentinova L, Beer NL, Stanik J et al: Identification and functional characterisation of novel glucokinase mutations causing maturity-onset diabetes of the young in Slovakia. PLoS One 2012; 7: e34541.

20 Bellanne-Chantelot C, Levy DJ, Carette C et al: Clinical characteristics and diagnostic criteria of maturity-onset diabetes of the young (MODY) due to molecular anomalies of the HNF1A gene. J Clin Endocrinol Metab 2011; 96: e1346-e1351.

21 Stride A, Hattersley AT: Different genes, different diabetes: lessons from maturityonset diabetes of the young. Ann Med 2002; 34: 207-216.

22 Becker F, van El CG, Ibarreta D et al: Genetic testing and common disorders in a public health framework: how to assess relevance and possibilities. Background Document to the ESHG recommendations on genetic testing and common disorders. Eur J Hum Genet 2011; 19(Suppl 1): S6-S44.

23 Aarden E, Van Hoyweghen I, Horstman K: The paradox of public health genomics: definition and diagnosis of familial hypercholesterolaemia in three European countries. Scand J Public Health 2011; 39: 634-639.

24 Shepherd M, Hattersley AT, Sparkes AC: Predictive genetic testing in diabetes: a case study of Multiple perspectives. Qual Health Res 2000; 10: 242-259.

25 Colclough K, Saint-Martin C, Timsit J, Ellard S, Bellanné-Chantelot C: Clinical utility gene card for: Maturity-onset diabetes of the young. Eur J Hum Genet 2014, e-pub ahead of print 12 February 2014; doi:10.1038/ejhg.2014.14.

26 Burton H, Cole T, Farndon P: Genomics in Medicine. Cambridge: PHG Foundation, 2012, ISBN 978-1-907198-10-6. 